\title{
III. Geburtshilflich-gynäkologische Mitteilungen aus anderen medizinischen Gesellschaften
}

Verein für inncre Medizin und Kinderkrankheiten Berlin. 4. VII.

(Berl. klin. Woch. 19. IX. S. 1140.) Blumenthal: Über das therapeutische Problem der bösartigen Geschwülste. - Halberstädter: Biologische und technische Fragen bei der Tiefenbestrahlung.

Bespr.: Lazarus, Bleich-röder, Levy, Dorn, Max Cohn, Przybílla.

Ärztlicher Verein München. 15. VI. (Berl. klin. Woch. 26. IX. S. 1166.) Scholten: Über Infusion und Transfusion. - Eisenreich: Über Indikationsstellung der geburtshilflichen Operationen. Hirsch: Mitteilungen über die Wirkung der obligatorischen Prophylaxe der Ophthalmoblenorrhoea Neonatorum. - Döderleín: Demonstration von geburtshilflichen Natur- und Kunstfilmen. - 6. VII. Zweifel, E. : K\ırze Mitteilungen über die Verbreitung und Häufigkeit des Karzinom. - A. Müller: Der Einfluß der Kindslagen auf die Kopfform der Kinder und der Erwachsenen sowie auf den Geburtsmechanismus der Kopfgeburten.

Köln, Allgemeiner ärztlicher Verein. 20. IV. (Munch, med. Woch. 5. VIII. S. 1000.) Herx: Uterusruptur und Darmverletzung. Bespr.: Fiith. - Filth: Fluor vagin. Bespr.: Ebeler. Heidelberg, Naturh.-med. Verein. 15. VII. (Munch, med. Woch. 23. IX. S. 1234.) Raefles: Hypnose in der Gynäkologie. Bespr.: Wich-mann, Meyer, Sachs, Ganz.

Freiburg, Med. Gesellschaft. 22. II. (Med. Klin. 11. IX. S. 1130.) Friedrich : Weitere experimentelle Untersuchungen über Sekundärstrahlen-Therapie.

Münchn. Ärztl. Verein. 1. VI. (Med. Klin. 25. IX. S. 1191.) v. d. Pfordten:

Sittlichkeitsverbrechen im neuen Strafgesetzentwurf.

Prag, Verein deutscher Irzte. 3. III. Med. Klin. 25. IX. S. 1191.) H. H. Sehmid: Parametranes Exsudat durch Gasbrandbazillus. - G. A. Wagner: Primäres Collumkarzinom, durch Ausschabung des Corpus entdeckt. nachdem es dieses ergriffen. - 10. VI. Weinzierl: Erfahrungen mit der Kaseosantherapie.

Greifswald, Medizinischer Verein. 24. VI. (Med. Klin. 9. X. S. 1249.) Stephan: Über die Bedeutung des Glykogens für die Biologie der Vagina.

München, Ärztlicher Verein. 15. VI. (Med. Klin. 2. X. S. 1218.) Scholten : Über Infusion und Transfusion. - Eisenreich : Über Indikationsstellung der geburtshilflichen Operationen. Zweifel: Kurze Mitteilungen über Verbreitung und Häufigkeit des Karzinom. Bespr.: Döderlein. - A. Müller: Der Einfluß der Kindslagen auf Kopfform der Kinder und der Erwachsenen sowie auf den Geburtsmechanismus der Kopfgeburten. Bespr.: Döderleín.

102 Greburtsh.-gynäkolog. Mitteilungen aus medizinischen Gesellscliaften.

Kònigsberg, Verein für wissenschaitliche Heilkunde. 9. Y. (Dtsch. med. Woch. 8. IX. S. 1077.) Klewitz: Uber Schädigungen des Bhites durch Röntgenstrahlen. Bespr.: Frohmann, Kirschner.

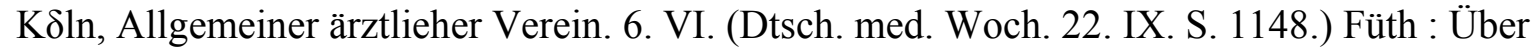
Wesen und Behandlung der Dysmenorrhöe. Bespr.: Koch, Ernst. Breslau, Ver. f. vaterl. Kultur. 10. VI. (Med. Klin. 23. X. S. 1311.) Weil: Die Ätiologie der Armlähmungen der Neugeborenen. Bespr.: Küstner, Liegher, Diehmann. 
Frankfurt a. M·, Ärztlieher Verein. 19. IX. (Med. Klin. 30. X. S. 1343.) Seitz: Klinische

Erfahrungen mit der Röntgenbestrahlung bösartiger T1/8noren. Bespr.: Flörcken.

Hamburg', Ärztlieher Verein. 17. V. (Med. Klin. 30. X. S. 1344.) Kiimmel, Behandlung von Karzinom durch Blutinjektionen. 20 Fälle, vorübergehende Besserung, kein wirklicher Erfolg. Prag, Verein deutscher Ärzte. 21. VI. (Med. Klin. 16. X. S. 1281.) H. H. Schmid, Röntgenbild eines verkalkten Myom, das einen Blasenstein vortäuscht.

Berlin, Ges. f. Chirurgie. 11. VI. (Dtsch. med. Woch. 13. X. S. 1243.) Martens: Ersatz der fehlenden Vagina durch Darm. Bespr.: Hinz, Hollander, Körte.

Berlin, Verein ï. innere Medizin und Kinderkrankheiten. 9. V. (Dtsch. med. Woch. 20. X. S. 1279.) Foth: Zur Xabeldiphtherie. Bespr.: David-sohn, Soldin, Cassel.

Freiburg i. Br., Mediz. Ges. 24. V. (Med. Klin. 6. XL S. 1372.) v. Möllendorf: Uber zwei rnenschliche Keime.

Dortmund, Klinische Demonstrationsabende. Mai 21. (Med. Klin. 6. XI. S. 1373.) Engelmann : Über die Notwendigkeit der Frühoperatioa der Eileiterschwangerschaft. - Über die Bedeutung der rektalen Unter-stichung in der Gebiirtshilfe und Gynäkologie. - Kl. Hoffmann : Über eine neue Geburtszange (Kielland-Zange).

Leipzig, Medizinische Ges. 28. VI. (Dtsch. med. Woch. 27. X. S. 1313. Zweifel: Die Erfolge der operativen Behandlung der malignen Tumoren des Uterus gegenüber denen der

Strahlenbehandlung.

Wien. med. Ges. Juli. (Dtsch. med. Woch. 27. X. S. 1314.) Heidler: Hypoplasie der linken

Mamma.

Hamburg-, Ärztl. Verein. 28. VI. (Med. Klin. 20. XI. S. 1430.) Calmann: Achsendrehung eines schwangeren Horns bei Uterus bicornis septus. Cum vagina septa.

Bonn, Niederrh. Ges. f. Natur- Heilk. 18. VII. (Dtsch. med. Woch. 17. XI. S. 1412.) Els:

Künstliche Harnblasenbildung nach der Methode von Makkas. - Freund: Demonstration einer nach Mokkas operierten Patientin. - Hinselmann: Einfluß cler Körperhaltung auf die Kapillarströmung.

Ein Jahrhundert Totalexstirpation usw. Buchbesprecliungen. 103

IV. Ein jahrhundert Totalexstirpation des karzinomatösen Uterus.

Am 28. Januar 1822 hat Joh. Nep. Santer in Konstanz die erste radikale Exstirpation des karzinomatösen Uterus ausgeführt. Die Kranke überstand die Operation - anscheinend mit einer Blasenscheidenfistel -, wie sich heransstellte, als sie 4 Monate später an einer interkurrenten Krankheit starb.

Vor Santer hatten Osianäer und Langeńbech der Ältere schon den pro-labierten Uterus subperitoneai ausgeschnitten. Santer unternahm die Operation an dem in situ befindlichen. Er versuchte ebenfalls eine solche Ausschälung, indem er Blase und Peritoneum mit dem Skalpellstiel abschabte. Er mußte dann das Peritoneum spalten und den Uterus vorn herauswälzen. Ohne Unterbindung zu machen, füllte er die Scheide mit aluminierter Charpie. Santers Bericht1) erfüllt den Leser mit tiefer Anerkennung · für den kühnen Operateur. Erst W. A. Frennâ hat 1878 der Operation die allgemeine Anerkennung geschaffen; ihm íolgten Cserny, Karl Schroder, Billroth und
A. Martin (Juni 1878).
A. Martin.

V.

Buchbesprechungen.

Lahm, W·, Radium- Tiefentherapie, das Problem der modemen Radium-behandlung des Krebses. Dresden und Leipzig 1921. Th. Steinkopf. 52 Seiten. Geheftet 5.- Mk. 
Nach einem einleitenden Vorworte Kehrers über die Geschichte und Eiitwicklung der modemen Radiumtiefentherapie und einer kurzen, Einleitung des Verf. bespricht er zuerst die theoretischen Grundlagen der Radiumtherapie, wie man den $\gamma$-Strahl des Radiums gewinnt, dessen Eigenschaften, um sich dann des genaueren mit der biologischen Wirkung des Radiums zu beschäftigen. Er schildert, wie Zellen und Gewebe auf die Einwirkung der Strahlen reagieren, und stellt den Begriff der Reiz-größe auf. Hierunter versteht er die lokale Rad.-El.-Konzentration mal Zeit, wobei unter lokaler Rad.-El.-Konzentration cliejenige Intensität zu verstehen ist, welche $1 \mathrm{mg}$ Rad.-El. in $1 \mathrm{~cm}$ Entfernung erzeugt. Hierauf bespricht er den Ablauf der biologischen Reaktion, um sich dann mit der zu verabfolgenden Dosis zu beschäftigen: Ovarial-, Karzinomdosis usw. In einem weiteren Abschnitte erörtert er des genaueren die Frage der Sekundärstrahlung; Streustrahlung, sekundäre Betastrahlung und Eigen-strahlung werden besprochen, sodann die Absorption und Härte der Strah-lung sowie das Quadratgesetz. In einem weiteren Abschnitte bespricht

x) Die gänzliche Exstírpation der karzinomatösen Gebärmutter ohne selbst entstandenen oder künstlich erzeugten Vorfall vorgenommen und glücklich vollführt von Dr. Joh. Nep. Santer, Großherzogl. Bad. Medizinal-rat und Amtsphysikus in Konstanz. Mit Abbildungen in Steindruck. Konstanz 1922. 